\title{
Conhecimento dos pacientes sobre os medicamentos prescritos após consulta médica e dispensação
}

\author{
Patient awareness about drugs prescribed \\ after medical appointment and prescription
}

Diony Oenning ${ }^{1}$

Bruna Volpato de Oliveira ${ }^{1}$

Carine Raquel Blatt ${ }^{2}$

${ }^{1}$ Acadêmicas, curso de Farmácia, Universidade do Sul de Santa Catarina Campus Tubarão. Avenida José Acácio Moreira 787, Dehon. 88704-900 Tubarão SC.

${ }^{2}$ Curso de Farmácia, Universidade do Sul de Santa Catarina Campus Tubarão.

carine.blatt@gmail.com

\begin{abstract}
Information provided to patients about medication is important for the success of the treatment, since the lack of such information is one of the main reasons why patients fail to take medicines as prescribed. The aim of this study was to verify patients' awareness about prescribed drugs distributed at the Grão Pará (Santa Catarina) primary healthcare unit after a medical appointment. One hundred and eleven patients were interviewed, seventy after a medical appointment and forty-one after drugs were distributed. $\mathrm{Pa}$ tient awareness was tested by asking questions about the name, usage, dosage, length of treatment, side effects, and precautions. After a medical appointment $28.5 \%$ of patients were classified as having a good level of awareness, $17.1 \%$ fair and $64.4 \%$ insufficient. After drugs were distributed $4.9 \%$ of patients were classified as having a good level of awareness, $87.8 \%$ fair and $7.3 \%$ insufficient. The majority of those interviewed had a low level of awareness about the use of the prescribed drugs. Results after drugs were distributed were better in comparison to results after a medical appointment, suggesting the importance of the pharmacist in informing patients about the correct use of drugs.
\end{abstract}

Key words Drugs, Drug utilization studies, Awareness, Rational use of drugs
Resumo As orientações sobre medicamentos fornecidas aos pacientes são fundamentais para o sucesso do tratamento, uma vez que a ausência delas é uma das principais causas do uso incorreto dos medicamentos. O objetivo deste estudo foi verificar o conhecimento dos pacientes sobre seu tratamento medicamentoso, após consulta médica e dispensação, realizadas na unidade básica de saúde do município de Grão Pará, em Santa Catarina. Foram entrevistados 111 indivíduos, 70 após a consulta médica e 41 após a dispensação dos medicamentos. O nivel de conhecimento foi verificado mediante perguntas relativas ao nome do medicamento, indicação, dose, frequência de uso, duração do tratamento, efeitos adversos e precauções. Após a consulta médica, 28,5\% foram classificados com nível bom de conhecimento; $17,1 \%$, regular; e 64,4\%, insuficiente. Após a dispensação, 4,9\% dos pacientes foram classificados com bom nivel de conhecimento; $87,8 \%$, regular; e 7,3\%, insuficiente. A maioria dos entrevistados não possui bom nível de informação para a administração de medicamentos. Os resultados encontrados após a dispensação foram melhores que os resultados encontrados após a consulta médica, o que sugere a importância do farmacêutico na orientação dos pacientes para a correta utilização dos medicamentos.

Palavras-chave Medicamentos, Estudos de utilização de medicamentos, Conhecimento, Uso racional de medicamentos 


\section{Introdução}

O uso racional de medicamentos, almejado pela Política Nacional de Medicamentos brasileira ${ }^{1}$, é o processo que compreende a prescrição apropriada, a disponibilidade oportuna e a preços acessíveis, a dispensação em condições adequadas e o consumo nas doses indicadas, nos intervalos definidos e no período de tempo determinado de medicamentos eficazes, seguros e de qualidade.

A implementação dessa prática objetiva melhorar o padrão de atendimento, colaborando significativamente com a redução de gastos. Ao contrário, o uso irracional de medicamentos pode causar malefícios à saúde dos usuários, tais como efeitos adversos, eficácia limitada, resistência a antibióticos e farmacodependência ${ }^{2,3}$. Entre os fatores que contribuem para o uso irracional de medicamentos não podemos deixar de destacar às estratégias de promoção e vendas das empresas farmacêuticas ${ }^{4,5}$ e o papel assumido pelo medicamento na atualidade ${ }^{6,7}$. Sendo que, este segundo, está em grande parte relacionado com o primeiro.

A promoção do uso racional dos medicamentos deve contar com a participação de diversos atores sociais: pacientes, profissionais de saúde, legisladores, formuladores de políticas públicas, indústria, comércio e governo ${ }^{8}$.

Os resultados disponibilizados pelos estudos de utilização de medicamentos podem contribuir com a instrumentalização do profissional para o reconhecimento da realidade cotidiana que envolve o uso de medicamentos ${ }^{9}$. Entre os principais problemas relacionados ao uso de medicamentos estão o sobreuso (como consequência da prescrição excessiva ou do uso indiscriminado), a polimedicação (prescrição de vários medicamentos) e o uso incorreto (medicamento ineficaz ou na dose errada $)^{2}$.

No Sistema Único de Saúde, o acesso aos medicamentos é mediado pela apresentação da prescrição, sendo esta uma ordem escrita dirigida ao farmacêutico, com a definição do medicamento que deverá ser fornecido ao paciente, bem como com a maneira que este deverá utilizá-lo. A prescrição constitui um documento legal pelo qual se responsabilizam quem prescreve (médico, dentista) e quem dispensa o medicamento (farmacêutico), ambos sujeitos à legislação de controle e a ações da vigilância sanitária ${ }^{10}$.

O profissional da saúde que dispensa medicamentos exerce um importante papel na utilização correta deles. A dispensação é uma das últimas oportunidades de identificar, corrigir ou re- duzir possíveis riscos à terapêutica medicamentosa, pois, além de dispensar o medicamento, de qualidade e de maneira correta, o farmacêutico deve complementar as informações passadas pelo médico ao paciente sobre os medicamentos prescritos, como os cuidados na administração e as orientações não farmacológicas, de forma a contribuir com seu uso racional e para a melhora do quadro clínico do paciente, sem o eventual aparecimento de efeitos indesejados para este ${ }^{2,11}$.

No processo de atenção à saúde, o paciente deve ser visto como membro ativo no processo saúde/doença/tratamento ${ }^{12}$. Dessa maneira, o paciente deve assumir solidariamente a responsabilidade por seu tratamento, uma vez que sua atitude interfere no sucesso da terapêutica. A importância do paciente se dá pelo fato de ser ele quem descreve o que está sentindo e de ser quem vai aderir ou não à indicação médica, pois, se ele não seguir as recomendações prescritas e não utilizar corretamente os medicamentos, na maioria das vezes, o tratamento não alcançará o objetivo esperado ${ }^{2,13}$.

Para que isso ocorra é necessário haver comunicação. O paciente tem direito às informações sobre sua saúde, medicamentos que deverá utilizar, objetivos e riscos de seu tratamento, sendo dever dos profissionais da saúde fazer com que tais informações cheguem a ele de forma clara e objetiva, para que possa seguir de maneira eficiente tais recomendações ${ }^{2,10}$.

A falta de informações ou a não-compreensão das informações transmitidas pelos profissionais da saúde aos pacientes podem trazer consequências como: não-adesão ao tratamento, com o consequente insucesso terapêutico; retardo na administração do medicamento, agravando o quadro clínico do paciente; aumento da incidência de efeitos adversos, por inadequado esquema de administração e/ou duração do tratamento; dificuldades na diferenciação entre manifestações da doença e efeitos adversos da terapêutica; e incentivo à automedicação, bem como outras sérias consequências, que podem piorar o estado de saúde do paciente ${ }^{10,14,15}$.

Considerando a problemática do uso irracional dos medicamentos e a importância da comunicação do prescritor e do dispensador com o paciente para a correta utilização deles, para o alcance dos objetivos terapêuticos, este estudo foi desenvolvido com o objetivo de medir o nível de conhecimento dos pacientes sobre os medicamentos após consulta médica e dispensação na unidade básica de saúde central do município de Grão Pará, em Santa Catarina. 


\section{Metodologia}

A pesquisa foi realizada no período de fevereiro a maio de 2007, no município de Grão Pará, o qual possui 6.273 habitantes ${ }^{16}$ e está localizado na região sul do estado de Santa Catarina.

No município existem três unidades básicas de saúde, duas localizadas no interior e uma unidade central. $\mathrm{O}$ atendimento aos pacientes na unidade central é realizado por uma equipe multiprofissional, composta de cinco médicos com especializações distintas, duas enfermeiras, quatro auxiliares de enfermagem, uma técnica de enfermagem, três dentistas e um farmacêutico.

Os pacientes incluídos neste estudo foram aqueles que, durante o período de coleta de dados, utilizaram os serviços de saúde da unidade básica central do município.

O nível de conhecimento dos pacientes sobre medicamentos após consulta e dispensação foi medido por um estudo transversal realizado em dois grupos de pacientes. No primeiro foram entrevistados os pacientes que realizaram consulta médica na unidade básica de saúde. No segundo foram entrevistados os pacientes que retiraram medicamentos da unidade básica de saúde. Os dois grupos foram entrevistados pelas autoras, em dias e períodos aleatórios.

Para o cálculo do tamanho da amostra utilizou-se a fórmula descrita por Medronho ${ }^{17}$ para estudos de prevalência, considerando um intervalo de confiança de $95 \%$ e um erro estimado de $15 \%$, o tamanho da amostra calculado foi de 43 pessoas para cada grupo pesquisado.

Quando o paciente era menor de 18 anos, o entrevistado era seu responsável. Foram excluídos os pacientes que não concordaram em assinar o termo de consentimento livre e esclarecido, os pacientes que não apresentaram receita médica e os pacientes menores de 18 anos desacompanhados de um responsável.

Os pacientes foram entrevistados pelas pesquisadoras com o suporte de um questionário estruturado com perguntas abertas e fechadas, previamente testado em estudo piloto realizado em uma unidade básica de saúde de um município vizinho.

O nível de conhecimento foi verificado mediante perguntas relativas ao nome do medicamento, indicação terapêutica, dose e frequência de administração, duração do tratamento, precauções e cuidados na administração, e efeitos adversos. Também foram verificados dados referentes à prescrição médica, às classes mais prescritas, ao número de medicamentos por receita, aos tempos médios de consulta e de dispensação, ao nível de satisfação com a consulta e com a dispensação, e se o paciente já fez uso do medicamento prescrito. Além disso, foram verificadas algumas características socioculturais do paciente, como sexo, idade e escolaridade.

Durante a entrevista, o paciente podia consultar a receita ou qualquer outro material para responder às questões relativas ao medicamento. Todas as perguntas foram direcionadas para o primeiro medicamento prescrito.

Seguindo a metodologia proposta por Silva et al. ${ }^{15}$, os dados foram classificados de acordo com o nível de informação dos entrevistados, partindo de uma definição segura do uso de medicamentos, de forma a não causarem danos à saúde do paciente. Foram definidos os níveis de informação: 1 - nível bom, o qual dá ao paciente condições seguras de usar os medicamentos, em qualquer hipótese; 2 - nível regular, que dá ao paciente condições de usar os medicamentos de maneira segura, sem nenhum tipo de variação no tratamento; e 3 - nível insuficiente, o qual não dá ao paciente condições de utilizar os medicamentos de forma segura.

Dessa forma, foram atribuídos dois pontos para cada um dos itens "nome do medicamento", "dose" e "frequência de administração", e um ponto para "duração do tratamento", "indicação terapêutica", "efeitos adversos" e "precauções", podendo-se alcançar um máximo de dez pontos. Pacientes que somaram menos de seis pontos foram classificados com nível de informação insuficiente; aqueles com seis a oito pontos foram classificados com nível de informação regular; e os com mais de oito pontos foram classificados com nível de informação bom.

O presente trabalho foi aprovado pelo Comitê de Ética da Universidade do Sul de Santa Catarina (Unisul).

\section{Resultados}

Foram entrevistados 111 pacientes, 70 após a consulta e 41 após a dispensação. A distribuição dos pacientes por sexo, idade e escolaridade média pode ser visualizada na Tabela 1 .

Dos entrevistados após a consulta médica, $6 \%$ eram os responsáveis pelo paciente, enquanto no grupo entrevistado após a dispensação os responsáveis perfaziam $20 \%$.

Todas as consultas médicas foram realizadas por clínico geral, $88 \%$ das dispensações foram realizadas por farmacêutico, e 12\%, por enfermeiro. 
$\mathrm{Na}$ Tabela 2 podem ser verificadas as informações sobre qual a duração média da consulta e da dispensação, se o paciente recebeu orientação para o uso da medicação e se o paciente ficou satisfeito com a consulta e com a dispensação. $\mathrm{O}$ número médio de medicamentos por receita foi de 1,2 $\pm 0,40$ no grupo entrevistado após consulta médica e de $1,1 \pm 0,37$ no grupo após dispensação. Registra-se que $86 \%$ dos pacientes receberam a prescrição de apenas um medicamento.

O conhecimento dos pacientes quanto aos medicamentos prescritos está descrito nas Tabelas 3 e 4.

Entre os entrevistados, 53\% do grupo após a consulta e $76 \%$ do grupo após a dispensação já haviam utilizado pelo menos uma vez o medicamento prescrito.

Tabela 1. Sexo, idade e escolaridade dos pacientes entrevistados após a consulta e a dispensação dos medicamentos na unidade básica de saúde do município de Grão Pará - SC, 2007.

\begin{tabular}{lcc}
\hline \multicolumn{1}{c}{ Variáveis } & $\begin{array}{c}\text { Consulta } \\
(\mathbf{n}=\mathbf{7 0})\end{array}$ & $\begin{array}{c}\text { Dispensação } \\
(\mathbf{n}=\mathbf{4 1})\end{array}$ \\
\hline Sexo feminino & $72,8 \%$ & $78,0 \%$ \\
Idade média (em anos) & $40,67 \pm 15,82$ & $43,46 \pm 15,01$ \\
Escolaridade (em anos) & $8,12 \pm 3,43$ & $7,08 \pm 3,14$ \\
\hline
\end{tabular}

Tabela 2. Tempo médio da consulta e da dispensação dos medicamentos na unidade básica de saúde do município de Grão Pará - SC, 2007.

\begin{tabular}{lcc}
\hline \multicolumn{1}{c}{ Variáveis } & $\begin{array}{c}\text { Consulta } \\
(\mathbf{n = 7 0})\end{array}$ & $\begin{array}{c}\text { Dispensação } \\
(\mathbf{n}=\mathbf{4 1})\end{array}$ \\
\hline $\begin{array}{l}\text { Tempo médio (em minutos) } \\
\text { Recebeu orientação de como }\end{array}$ & $5,94 \pm 1,82$ & $2,60 \pm 1,03$ \\
utilizar os medicamentos & & \\
Sim & $60,0 \%$ & $92,7 \%$ \\
Sim, mas não entendeu & $10,0 \%$ & $4,9 \%$ \\
Não & $30,0 \%$ & $2,4 \%$ \\
Nível de satisfação & & \\
Ótimo & $1,4 \%$ & $7,3 \%$ \\
Bom & $85,7 \%$ & $87,8 \%$ \\
Regular & $12,9 \%$ & $4,9 \%$ \\
\hline
\end{tabular}

\section{Discussão}

No grupo pesquisado após a dispensação o número de entrevistados foi de 41, uma vez que, durante o período de coleta de dados, a maioria dos medicamentos estava em falta na Unidade Básica de Saúde e o número de pacientes que retiraram os medicamentos ficou reduzido.

Nos dois grupos verificou-se a prevalência de mulheres com média de idade acima de 40 anos e com 7 anos de escolaridade. Quanto ao número médio de medicamentos prescritos, com o qual se pode avaliar o grau de polimedicação dos pacientes, o valor encontrado com a realização do estudo foi de 1,2 medicamento por paciente, que é menor em comparação com valores encontrados em estudos anteriores ${ }^{18,19}$, cuja média foi de 2,0 e 2,3 medicamentos por prescrição.

Tabela 3. Percentual de respostas corretas sobre o nome do medicamento prescrito, indicação terapêutica, dose e frequência de administração, duração de tratamento, precauções e cuidados na administração e efeitos adversos após a consulta e a dispensação dos medicamentos na unidade básica de saúde do município de Grão Pará - SC, 2007.

\begin{tabular}{|c|c|c|}
\hline Variáveis & $\begin{array}{c}\text { Consulta } \\
(\mathbf{n}=70)\end{array}$ & $\begin{array}{c}\text { Dispensação } \\
(n=41)\end{array}$ \\
\hline Nome do medicamento & 40,0 & 92,7 \\
\hline Indicação terapêutica & 95,7 & 97,6 \\
\hline $\begin{array}{l}\text { Dose e frequência de } \\
\text { administração }\end{array}$ & 60,0 & 92,7 \\
\hline Duração do tratamento & 38,6 & 82,9 \\
\hline $\begin{array}{l}\text { Precauções e cuidados } \\
\text { na administração }\end{array}$ & 11,4 & 4,9 \\
\hline Efeitos adversos & 0,0 & 0,0 \\
\hline
\end{tabular}

Tabela 4. Nível de conhecimento dos pacientes quanto a utilização dos medicamentos, após a consulta e dispensação dos medicamentos na unidade básica de saúde do município de Grão Pará - SC, 2007.

\begin{tabular}{lcc}
\hline & $\begin{array}{c}\text { Consulta } \\
(\mathbf{n = 7 0})\end{array}$ & $\begin{array}{c}\text { Dispensação } \\
(\mathbf{n}=\mathbf{4 1})\end{array}$ \\
\hline Bom ( $\geq$ 8 pontos) & $28,5 \%$ & $4,9 \%$ \\
Regular (6 a 8 pontos) & $17,1 \%$ & $87,8 \%$ \\
Insuficiente (<6 pontos) & $64,4 \%$ & $7,3 \%$ \\
\hline
\end{tabular}


O tempo médio de consulta obtido no estudo foi de 5,94, inferior a outros estudos similares, cujo tempo médio de consultas foi de 9,2 minutos $^{20}$ e 9,4 minutos ${ }^{19}$. Segundo a Portaria no 3.046 do Ministério da Saúde e recomendação da OMS, o tempo sugerido para que a consulta seja adequadamente realizada é de 15 minutos ${ }^{20}$. O pouco tempo de consulta pode significar baixa qualidade do atendimento, por possivelmente omitir etapas importantes, como a anamnese, a solicitação de exames diagnósticos, a formulação de um tratamento farmacológico e/ou não farmacológico e a correta orientação para o uso da medicação, de modo que o problema de saúde seja resolvido ou controlado. Por outro lado, o pouco tempo de consulta também pode ser explicado pela elevada procura dos serviços oferecidos, o que inviabiliza um atendimento mais racional e humanizado ${ }^{21}$.

Com relação à dispensação dos medicamentos, a OMS preconiza que o farmacêutico destine à orientação ao menos três minutos por pacien$\mathrm{te}^{20}$. O tempo médio de dispensação obtido no estudo foi de 2,6 minutos por paciente, superior à média de outros estudos. No estudo realizado em 10 unidades de saúde de Ribeirão Preto, o tempo médio de dispensação foi de 18,4 segundos ${ }^{20}$; em outro, realizado em 15 centros de saúde do Distrito Federal, esse tempo foi de 52,3 segundos ${ }^{19}$.

Pressupõe-se que, quanto maior o tempo de contato entre o prescritor e o paciente ou entre o dispensador e o paciente, mais tempo é dedicado à transmissão de informações entre os atores, o que contribui para o maior entendimento do paciente sobre a correta utilização dos medicamentos.

Embora $70 \%$ e $98 \%$ dos entrevistados afirmaram ter recebido orientação de como utilizar o medicamento durante a consulta e a dispensação, respectivamente, os resultados de avaliação do nível de conhecimento dos pacientes acerca dos medicamentos foi regular ou insuficiente. Isso sugere que ou o tempo de orientação é insuficiente ou as informações não estão sendo repassadas adequadamente.

Um ponto a se destacar é que, mesmo sem conhecer as informações para o correto uso dos medicamentos, mais de $85 \%$ dos pacientes estavam satisfeitos com a consulta e com a dispensação. Tal fato pode estar relacionado com o nãoreconhecimento de que o uso incorreto do medicamento pode trazer danos à saúde, e também com a valorização de outros aspectos, como a atenção recebida pelo paciente.

De acordo com a classificação do nível de conhecimento dos usuários sobre os medicamen- tos, foi possível verificar o baixo nível de conhecimento para a garantia do uso correto dos medicamentos, uma vez que $64 \%$ dos entrevistados após a consulta médica foram classificados com nível insuficiente de informação, ou seja, não apresentavam condições de utilizar os medicamentos de maneira segura. Após a dispensação, $88 \%$ foram classificados com conhecimento regular.

Considerando que todos os entrevistados tinham a possibilidade de consultar a receita médica para responder ao questionário e que a maioria deles já havia utilizado o medicamento, o percentual de pacientes que não sabia quando e como deveria usá-lo é bastante expressivo. Esse fato é relevante, tendo em vista que essas informações são imprescindíveis ao cumprimento do esquema terapêutico estabelecido pelo médico.

Para todas as variáveis consideradas na avaliação do nível de conhecimento sobre os medicamentos, os valores de resposta correta foram maiores no grupo após a dispensação. Considerando que quase $90 \%$ das dispensações foram realizadas por farmacêutico, tal dado aponta para a importância deste no processo de atenção à saúde, por orientar o paciente sobre o uso correto do medicamento, de maneira a resolver ou a controlar o problema de saúde do paciente e a evitar problemas relacionados ao uso incorreto.

Em uma revisão dos estudos de intervenção do farmacêutico no uso de medicamentos por pacientes idosos ${ }^{22}$, de uma forma geral, as intervenções realizadas por farmacêuticos mostram resultados positivos, ao reduzir custos, melhorar as prescrições, promover maior adesão do paciente ao tratamento e controlar a possibilidade de reações adversas.

Quanto às informações, os melhores resultados foram encontrados para a indicação terapêutica, com mais de $95 \%$ de respostas corretas, talvez por ser esse o motivo de sua procura pelo serviço de saúde.

Após a consulta, menos de $40 \%$ conheciam o nome do medicamento prescrito e a duração do tratamento, e $60 \%$ conheciam a dose e a frequência de administração.

A falta da definição do tempo de tratamento pode ocasionar problemas ao paciente, devido tanto ao uso prolongado quanto por período insuficiente para o sucesso do tratamento. A nãoidentificação do nome do medicamento, mesmo tendo-se a possibilidade de consultar a receita, pode levar a confusões e à troca do medicamento.

O presente estudo evidenciou que, durante a consulta e a dispensação, os pacientes não foram informados, ou foram mal informados, sobre 
precauções e cuidados na administração do medicamento e efeitos adversos, o que é corrobora um estudo ${ }^{15}$ que fez uma entrevista com 264 sujeitos, dos quais apenas $36 \%$ responderam conhecer os cuidados que deveriam ter na administração do medicamento e somente $20 \%$ conheciam os possíveis efeitos adversos. A insuficiência de informações com respeito aos efeitos adversos pode estar relacionada à valorização secundária dada a esse item da informação, tanto por quem fornece a prescrição e o medicamento, quanto por quem o utiliza ${ }^{15}$. A possibilidade de o medicamento causar efeitos adversos acaba sendo, muitas vezes, omitida pelo prescritor, com receio de que informações negativas relativas ao medicamento possam prejudicar a adesão do paciente ao tratamento ou mesmo que, por autossugestão, o paciente venha a sentir o efeito adverso mencionado.

\section{Conclusão}

Diante dos resultados obtidos, conclui-se que a maioria dos entrevistados não possui bom nível de informação para a administração de medicamentos. Muitos deles tomam o medicamento sem ao menos saber para que serve, como administrá-lo corretamente e por quanto tempo.

Nesse sentido, é preciso que sejam aprimorados os meios de fornecer a informação ao paciente. Isso envolve a formação dos profissionais, a reorganização do tempo dedicado à orientação pelo prescritor e à atuação direta do farmacêutico na dispensação dos medicamentos, bem como à organização dos serviços para que essas informações possam ser repassadas ao paciente.

Por fim, resultados melhores foram encontrados após a dispensação dos medicamentos, o que indica a importância da presença do profissional farmacêutico na orientação dos pacientes para a correta utilização dos medicamentos.

\section{Colaboradores}

D Oenning e BV Oliveira foram responsáveis pela coleta e análise de dados, bem como pela redação inicial do artigo. CR Blatt foi responsável pela orientação da pesquisa e redação final do artigo. 


\section{Referências}

1. Brasil. Ministério da Saúde. Secretaria de Políticas de Saúde. Departamento de Atenção Básica. Política nacional de medicamentos. Brasília: Ministério da Saúde; 2001.

2. Marin N, organizadora. Assistência farmacêutica para gerentes municipais. Rio de Janeiro: OPAS, OMS; 2003.

3. Rozenfeld S. Prevalência, fatores associados e mau uso de medicamentos entre os idosos: uma revisão. Cad Saude Publica 2003; 19(3):717-724.

4. Angell M. A verdade sobre os laboratórios farmacêuticos: como somos enganados e o que podemos fazer a respeito. Rio de Janeiro: Record; 2007.

5. Barros JAC. Políticas farmacéuticas: a servicio de los interesses de la salud? Brasília: UNESCO/ANVISA; 2004.

6. Barros JAC. Nuevas tendencias de la medicalización. Cien Saude Colet 2008; 13(Supl.):579-587.

7. Lefevre F. O medicamento como mercadoria simbólica. São Paulo: Cortez Editora; 1991.

8. Aquino DS. Por que o uso racional de medicamentos deve ser uma prioridade? Cien Saude Colet 2008; 13(Supl.):733-736.

9. Leite SN, Vieira M, Veber AP. Estudos de utilização de medicamentos: uma síntese de artigos publicados no Brasil e América Latina. Cien Saude Colet 2008; 13(Supl.):793-802.

10. Wannmacher L, Ferreira MBC. Farmacologia clinica para dentistas. $2^{a}$ ed. Rio de Janeiro: Guanabara Koogan; 1999.

11. Vieira FS. Possibilidades de contribuição do farmacêutico para a promoção da saúde. Cien Saude Colet 2007; 12(1):213-220.

12. Leite SN, Vasconcellos MPC. Adesão à terapêutica medicamentosa: elementos para a discussão de conceitos e pressupostos adotados na literatura. Cien Saude Colet 2003; 8(3):775-782.

13. Pepe VLE, Castro CGSO. Interação entre prescritores, dispensadores e pacientes: informação compartilhada como possível beneficio terapêutico. Cad Saude Publica 2000; 16(3):815-822.

14. Lage EA, Freitas MIF, Acurcio FA. Informação sobre medicamentos na imprensa: uma contribuição para o uso racional? Cien Saude Colet 2005; 10(Supl.):133139.
15. Silva T, Schenkel EP, Mengue SS. Nível de informação a respeito de medicamentos prescritos a pacientes ambulatoriais de hospital universitário. Cad Saude Publica 2000; 16(2):449-455.

16. Data SUS. Caderno de Informação em Saúde. [acessado 2009 jun 24]. Disponível em: http://tabnet. datasus.gov.br/tabdata/cadernos/SC/SC_Grao_ Para_Geral.xls.

17. Medronho RA, organizador. Epidemiologia. São Paulo: Atheneu; 2006.

18. Girotto E, Silva PV. A prescrição de medicamentos em um município do Norte do Paraná. Rev Bras Epidemiol 2006; 9(2):226-234.

19. Naves JOS, Silver LD. Evaluation of pharmaceutical assistance in public primary care in Brasília, Brazil. Rev Saúde Pública 2005; 39(2):223-230.

20. Santos V, Nitrini SMOO. Indicadores do uso de medicamentos prescritos e de assistência ao paciente de serviços de saúde. Rev Saude Publica 2004; 28(6):819-834.

21. Arrais PSD, Barreto ML, Coelho HLL. Aspectos dos processos de prescrição e dispensação de medicamentos na percepção do paciente: estudo de base populacional em Fortaleza, Ceará, Brasil. Cad Saude Publica 2007; 23(4):927-937.

22. Romano-Lieber NS. Revisão dos estudos de intervenção do farmacêutico no uso de medicamentos por pacientes idosos. Cad Saude Publica 2002; 18(6): 1499-1507.

Artigo apresentado em 29/06/2009

Aprovado em 17/08/2009

Versão final apresentada em 12/11/2009 